\title{
DISCRETIZATION OF BAKER-AKHIEZER MODULES AND COMMUTING DIFFERENCE OPERATORS IN SEVERAL DISCRETE VARIABLES
}

\author{
ANDREY MIRONOV AND ATSUSHI NAKAYASHIKI \\ Dedicated to Viktor Matveevich Buchstaber \\ on his seventieth birthday
}

\begin{abstract}
We introduce the notion of discrete Baker-Akhiezer (DBA) modules, which are modules over the ring of difference operators, as a discretization of BakerAkhiezer modules, which are modules over the ring of differential operators. We use it to construct commuting difference operators with matrix coefficients in several discrete variables.
\end{abstract}

\section{INTRODUCTION}

In this paper we introduce the notion of discrete Baker-Akhiezer modules and, with the help of them, construct commutative rings of difference operators with matrix coefficients in several discrete variables and with spectral parameter from certain algebraic varieties.

We first recall some basic facts on commuting difference operators in one variable. Common eigenfunctions of two commuting difference operators,

$$
L_{1}=\sum_{i=N_{-}}^{N_{+}} v_{i}(n) T^{i}, \quad L_{2}=\sum_{i=M_{-}}^{M_{+}} u_{i}(n) T^{i},
$$

are parametrized by points of some algebraic curve $\Gamma$ :

$$
L_{1} \psi(n, P)=\lambda(P) \psi(n, P), L_{2} \psi(n, P)=\mu(P) \psi(n, P) .
$$

Krichever and Novikov 1 proved that there are points $P_{1}, \ldots, P_{k}$ on $\Gamma$ such that the whole commutative ring of difference operators, containing $L_{1}$ and $L_{2}$, is isomorphic to the ring of meromorphic functions with poles only at $P_{1}, \ldots, P_{k}$. In the case $k=2$ (two-point construction) explicit forms of operators were found in 2, 3. The theory of $n$-point operators was developed in [1]. Krichever and Novikov classified one-point operators of rank $l$ and found operators of rank two corresponding to the spectral curve of genus one. The theory of such operators is connected with the theory of higher rank algebro-geometric solutions of the 2D-Toda chain 1 1. In 4 Krichever-Novikov operators with polynomial coefficients are found.

In the case of operators, either differential or difference, of several variables, there has been no classification theorem up to now (for some results in this direction see [5, 6, 7]). The main difficulty is as follows. If ordinary difference operators (1) have a family of common eigenfunctions parametrized by an algebraic curve with $\lambda$ and $\mu$ being functions on it, then they commute. On the other hand, in the case of operators of several variables, the existence of a big family of common eigenfunctions is not enough for commutativity. For example, it is not difficult to construct operators possessing a family of common eigenfunctions parametrized by points of an algebraic variety which do not commute. This is a major difference between one and higher dimensional cases. 
Then the main question is how many common eigenfunctions are enough for the commutativity in the multi-dimensional case. An answer to this question is partially given in the papers of the second author 8, 9. In these papers the notion of a Baker-Akhiezer (BA) module over the ring of differential operators is introduced. It allows one to obtain commuting differential operators in several variables with matrix coefficients.

In this paper we introduce a discrete analogue of BA modules. This makes it possible to construct commuting partial difference operators with matrix coefficients as an analogue of the construction of commuting differential operators.

Definition 1. Let $X$ be an algebraic variety and $Y$ a subvariety of $X$. Then the set of $\mathbb{C}$-valued functions $\hat{M}=\left\{\psi(n, P) \mid n \in \mathbb{Z}^{g}, P \in X\right\}$ is called a DBA module if the following conditions are satisfied:

1. $T_{i} \psi(n, P) \in \hat{M}$, where $T_{i}$ is a shift operator on the $i$-th discrete variable of $n=$ $\left(n_{1}, \ldots, n_{g}\right)$.

2. $f(n) \psi(n, P) \in \hat{M}$, for an arbitrary function $f(n)$ from a certain class.

3. $\lambda(P) \psi(n, P) \in \hat{M}$ for each meromorphic function $\lambda(P)$ on $X$ having poles only on $Y$.

4. The sum of any two elements of $\hat{M}$ belongs to $\hat{M}$.

Let $A_{Y}$ be the ring of meromorphic functions on $X$ with poles only on $Y$ and $\mathcal{T}_{g}=$ $\hat{\mathcal{K}}\left[T_{1}, \ldots, T_{g}\right]$ be the ring of difference operators, where $\hat{\mathcal{K}}$ is a ring of certain functions on $\mathbb{Z}^{g}$. Properties 1-3 imply that $\hat{M}$ is a module over $\mathcal{T}_{g}$ and, at the same time, over $A_{Y}$. We call $\hat{M}$ a discrete Baker-Akhiezer (DBA) module.

Suppose that $\hat{M}$ is a free $\mathcal{T}_{g}$-module of finite rank. Then the DBA module allows us to construct commuting difference operators in several variables. Indeed, let us choose a free basis $\psi_{1}, \ldots, \psi_{N}$ in $\hat{M}$ and consider the vector-valued function $\Psi={ }^{t}\left(\psi_{1}, \ldots, \psi_{N}\right)$. Then for $\lambda \in A_{Y}$ there exists a uniquely defined difference operator $D(\lambda)$ with matrix coefficients such that

$$
D(\lambda) \Psi=\lambda \Psi
$$

since $\hat{M}$ is a free $\mathcal{T}_{g}$-module. Similarly, for $\mu \in A_{Y}$, we have

$$
D(\mu) \Psi=\mu \Psi .
$$

Operators $D(\lambda)$ and $D(\mu)$ commute, since $\hat{M}$ is free and $\lambda, \mu$ do not depend on the discrete variable $n$. This means that the family $\{\Psi(n, P)\}$ of common eigenvector-valued functions parametrized by points of $X$ is large enough, and hence the commutativity of these operators on the whole set of vector-valued functions follows from the commutativity on $\{\Psi(n, P)\}$.

We construct examples of free DBA modules of finite rank and commuting difference operators from abelian varieties with non-singular theta divisors and certain rational varieties as discretizations of the corresponding BA modules. We show that a basis of a BA module gives a basis of the corresponding DBA module. The situation when solutions of a continuous system directly give solutions of the corresponding discrete system is well known in soliton equations [10, 11.

The present paper is organized as follows. In section 2 we construct DBA modules explicitly and give main theorems. The DBA modules are defined as certain discretizations of Baker-Akhiezer $\mathcal{D}$ modules. Proofs of theorems are given in section 3 . In section 4 we give examples of explicit forms of commuting operators. 


\section{Construction of free DBA modules}

In this section we give two examples of free DBA modules which are constructed from Abelian varieties and certain rational varieties. In the first case elements of DBA modules and coefficients of difference operators are expressed in terms of theta functions, and in the second case the corresponding objects are expressed by elementary functions. All theorems in this section can be proved using the results on their differential analogues. The proofs themselves are given in section 3 .

2.1. DBA modules on Abelian varieties. Let $\tau$ be a point of the Siegel upper half space, $\theta_{a, b}(z, \tau)$ the Riemann's theta function with the characteristic ${ }^{t}\left({ }^{t} a,{ }^{t} b\right), a, b \in \mathbb{R}^{g}$, $X=\mathbb{C} /\left(\mathbb{Z}^{g}+\tau \mathbb{Z}^{g}\right), \Theta \subset X$ the theta divisor specified by the zero set of $\theta(z):=\theta_{0,0}(z, \tau)$ and $\mathcal{L}_{c}, c \in \mathbb{C}^{g}$, the flat line bundle on $X$ for which $\theta(z+c) / \theta(z)$ is a meromorphic section. A meromorphic section of $\mathcal{L}_{c}$ is identified with a meromorphic function $f(z)$ on $\mathbb{C}^{g}$ satisfying the condition

$$
f(z+m+\tau n)=\exp \left(-2 \pi i^{t} n c\right) f(z)
$$

for any $m, n \in \mathbb{Z}^{g}+\tau \mathbb{Z}^{g}$.

Let $L_{c}(m)$ be the space of meromorphic sections of $\mathcal{L}_{c}$ with poles only on $\Theta$ of order at most $m$ and $L_{c}=\bigcup_{m=0}^{\infty} L_{c}(m)$. A basis of $L_{c}(m)$ is given quite explicitly. Namely, for a nonnegative integer $m$ and $a \in \mathbb{Z}^{g} / m \mathbb{Z}^{g}$ we set

$$
F_{m, a}(z, c)=\theta_{a / m, 0}(m z+c, m \tau) / \theta(z)^{m} .
$$

Then the set of functions $\left\{F_{m, a}(z, c)\right\}$ is a basis of $L_{c}(m)$.

We denote by $\mathcal{K}$ the ring of meromorphic functions on $\mathbb{C}^{g}$. We denote the variable of a function of $\mathcal{K}$ by $x=\left(x_{1}, \ldots, x_{g}\right)$. Define the space $M_{c}$ by

$$
M_{c}=\bigcup_{m=0}^{\infty} M_{c}(m), \quad M_{c}(m)=\sum_{a} \mathcal{K} F_{m, a}(z, c+x) .
$$

This is nothing but the underlying space of the Baker-Akhiezer module of $(X, \Theta)[8$. We shall discretize it as follows.

For a function $F(z, x)$ define the operator $T_{i}$ by

$$
T_{i} F(z, x)=F\left(z, x+h_{i} e_{i}\right) \frac{\theta\left(z-h_{i} e_{i}\right)}{\theta(z)}, \quad F(z, x) \in M_{c},
$$

where $e_{i}$ is the $i$-th unit vector of $\mathbb{C}^{g}$ and $h_{i} \in \mathbb{C}$ is a parameter. It is easy to see that $T_{i}$ acts on $M_{c}$, since it preserves the relation (2) for $L_{c+x}$.

For $f(x) \in \mathcal{K}$ we associate the map $\hat{f}: \mathbb{Z}^{g} \rightarrow \mathcal{K}$ by

$$
\hat{f}(n)=f(x+n h),
$$

where $n=\left(n_{1}, \ldots, n_{g}\right)$ and $n h=\left(n_{1} h_{1}, \ldots, n_{g} h_{g}\right)$. We identify the map $\hat{f}$ with its value $\hat{f}(n)$. Let

$$
\hat{\mathcal{K}}=\{\hat{f}(n) \mid f \in \mathcal{K}\} .
$$

The space $\mathcal{K}$ naturally becomes a ring which we consider the ring of discrete functions with the discrete variable $n \in \mathbb{Z}^{g}$.

For a nonnegative integer $m$ and $a \in \mathbb{Z}^{g} / m \mathbb{Z}^{g}$ we define the map $\hat{F}_{m, a}: \mathbb{Z}^{g} \rightarrow M_{c}$ by

$$
\hat{F}_{m, a}(n)=T^{n} F_{m, a}(z, c+x),
$$

where $T^{n}=T_{1}^{n_{1}} \cdots T_{g}^{n_{g}}$. We identify the map $\hat{F}_{m, a}$ and its value $\hat{F}_{m, a}(n)$. We write $\hat{F}_{m, a}(n, z)$ if it is necessary to indicate the dependence on the variable $z$. 
Now we define the discrete Baker-Akhiezer module $\hat{M}_{c}$ by

$$
\hat{M}_{c}=\bigcup_{m=0}^{\infty} \hat{M}_{c}(m), \quad \hat{M}_{c}(m)=\sum_{a \in \mathbb{Z}^{g} / m \mathbb{Z}^{g}} \hat{\mathcal{K}} \hat{F}_{m, a}(n) .
$$

Explicitly,

$$
\hat{M}_{c}(m)=\sum_{a} \hat{\mathcal{K}} \frac{\theta_{a / m, 0}(m z+c+x+n h, m \tau)}{\theta(z)^{m}} \prod_{j=1}^{g}\left(\frac{\theta\left(z-h e_{j}\right)}{\theta(z)}\right)^{n_{j}} .
$$

We give an example of the elements in $\hat{M}_{c}(m)$ with $m=1,2$.

\section{Example.}

$$
\begin{gathered}
\frac{\theta(z+c+x+n h)}{\theta(z)} \prod_{j=1}^{g}\left(\frac{\theta\left(z-h e_{j}\right)}{\theta(z)}\right)^{n_{j}} \in \hat{M}_{c}(1), \\
\frac{\theta(z+c+x+n h+\beta) \theta(z-\beta)}{\theta^{2}(z)} \prod_{j=1}^{g}\left(\frac{\theta\left(z-h e_{j}\right)}{\theta(z)}\right)^{n_{j}} \in \hat{M}_{c}(2),
\end{gathered}
$$

where $\beta$ is an arbitrary constant from $\mathbb{C}^{g}$. The first example corresponds to $m=1, a=0$ in (3).

The operator $T_{i}$ acts on $\hat{M}_{c}$ as the shift operator:

$$
T_{i}\left(\hat{f}(n) \hat{F}_{m, a}(n)\right)=\hat{f}\left(n+e_{i}\right) \hat{F}_{m, a}\left(n+e_{i}\right) .
$$

Let $\mathcal{T}_{g}=\hat{\mathcal{K}}\left[T_{1}, \ldots, T_{g}\right]$ be the ring of difference operators with the coefficients in $\hat{\mathcal{K}}$. Then $\hat{M}_{c}$ becomes a $\mathcal{T}_{g}$-module.

Let $A=L_{0}$ be the ring of meromorphic functions on $X$ having poles only on $\Theta$. Obviously the space $L_{c+x}$ is an $A$-module. It follows that the $\operatorname{ring} A$ also acts on $\hat{M}_{c}$. In fact, for $f(z) \in A$, we have

$$
f(z) F_{m, a}(z, c+x)=\sum_{m^{\prime}, a^{\prime}} f_{m^{\prime}, a^{\prime}}(x) F_{m^{\prime}, a^{\prime}}(z, c+x),
$$

for some $f_{m^{\prime}, a^{\prime}}(x) \in \mathcal{K}$, since $L_{c+x}$ is an $A$-module. Notice that the multiplication by $f(z)$ commutes with the action of $T_{i}$. Therefore, applying $T^{n}$ to (41), we have

$$
f(z) \hat{F}_{m, a}(n)=\sum_{m^{\prime}, a^{\prime}} \hat{f}_{m^{\prime}, a^{\prime}}(n) \hat{F}_{m^{\prime}, a^{\prime}}(n)
$$

which shows that $f(z) \hat{M}_{c} \subset \hat{M}_{c}$. Consequently, $\hat{M}_{c}$ is a $\left(\mathcal{T}_{g}, A\right)$ bi-module.

In the following we assume that $\Theta$ is nonsingular. Then our first theorem is

Theorem 1. For an uncountable number of $h \in\left(\mathbb{C}^{*}\right)^{g}$ the module $\hat{M}_{c}$ is a free $\mathcal{T}_{g}$-module of rank $g$ !, where $\mathbb{C}^{*}=\mathbb{C} \backslash\{0\}$.

Corollary 1. For values of $h$ specified in Theorem 1 there exists a ring mono-morphism

$$
A \rightarrow \operatorname{Mat}\left(g !, \mathcal{T}_{g}\right)
$$


2.2. DBA-modules on rational varieties. We construct a rational spectral variety $\Gamma$ from $\mathbb{C} P^{1} \times \mathbb{C} P^{g-1}$ by identifying two hypersurfaces. In general we denote a point of the projective space $\mathbb{C} P^{m-1}$ by $\left[t_{1}, \ldots, t_{m}\right]$, while a point of the $m$ dimensional affine space is denoted by $\left(t_{1} \ldots, t_{m}\right)$.

Let us fix $a_{1}, a_{2}, b_{1}, b_{2} \in \mathbb{C}$ such that $\left(a_{i}, b_{i}\right) \neq(0,0)$ and $\left[a_{1}, b_{1}\right] \neq\left[a_{2}, b_{2}\right]$. Let $\mathcal{P}$ be a nondegenerate linear map $\mathcal{P}: \mathbb{C}^{g} \rightarrow \mathbb{C}^{g}$, and $\lambda_{j}$ and $v_{j}, j=1 \ldots, g$, be the eigenvalues and the eigenvectors of $\mathcal{P}$ respectively. We assume that $\lambda_{i} \neq \lambda_{j}$ for $i \neq j$. Denote the induced map $\mathbb{C} P^{g-1} \rightarrow \mathbb{C} P^{g-1}$ by the same symbol $\mathcal{P}$.

We set

$$
\Gamma=\mathbb{C} P^{1} \times \mathbb{C} P^{g-1} /\left\{\left(\left[a_{1}, b_{1}\right], t\right) \sim\left(\left[a_{2}, b_{2}\right], \mathcal{P}(t)\right), t \in \mathbb{C} P^{g-1}\right\} .
$$

Then on $\Gamma$ there is a structure of an algebraic variety [12].

Let $f(P), f_{i}(P), i=1, \ldots, g$, be the function of $P=\left(z_{1}, z_{2}, t_{1}, \ldots, t_{g}\right) \in \mathbb{C}^{g+2}$ of the form

$$
\begin{gathered}
f\left(z_{1}, z_{2}, t_{1}, \ldots, t_{n}\right)=\sum_{k=1}^{g}\left(\alpha_{k} z_{1} t_{k}+\beta_{k} z_{2} t_{k}\right), \alpha_{k}, \beta_{k} \in \mathbb{C}, \\
f_{i}\left(z_{1}, z_{2}, t_{1}, \ldots, t_{g}\right)=\sum_{k=1}^{g}\left(\alpha_{i k} z_{1} t_{k}+\beta_{i k} z_{2} t_{k}\right), \alpha_{i k}, \beta_{i k} \in \mathbb{C} .
\end{gathered}
$$

Proposition 1 (12). For generic $(\alpha, \beta) \in \mathbb{C}^{2 g}$ and generic $\left(\alpha_{i}, \beta_{i}\right) \in \mathbb{C}^{2 g}, i=1, \ldots, g$, there exist $A, c_{1}, \ldots, c_{g} \in \mathbb{C}^{*}$ such that for every $t=\left(t_{1}, \ldots, t_{g}\right) \in \mathbb{C}^{g}$ the functions (5), (6) satisfy the following equations:

$$
\begin{aligned}
f\left(a_{1}, b_{1}, v_{j}\right) & \neq 0, \quad 1 \leq j \leq g, \\
f\left(a_{1}, b_{1}, t\right)-A f\left(a_{2}, b_{2}, \mathcal{P}(t)\right) & =0, \\
f_{i}\left(a_{1}, b_{1}, t\right)-c_{i} f_{i}\left(a_{2}, b_{2}, \mathcal{P}(t)\right) & =0, \quad 1 \leq i \leq g .
\end{aligned}
$$

According to (8) the equation

$$
f\left(z_{1}, z_{2}, t_{1}, \ldots, t_{g}\right)=0
$$

correctly defines a hypersurface in $\Gamma$.

For any $\Lambda \in \mathbb{C}^{*}$ the discrete Baker-Akhiezer module $\hat{M}_{\Lambda}$ is similarly defined to the case of Abelian varieties as the discretization of the Baker-Akhiezer module constructed in [12. It is defined directly by

$$
\begin{aligned}
& \hat{M}_{\Lambda}=\bigcup_{k=0}^{\infty} \hat{M}_{\Lambda}(k), \\
& \hat{M}_{\Lambda}(k)=\left\{\psi(n, P)=\frac{h(n, P)}{f(P)^{k}} \prod_{j=1}^{g}\left(\frac{f_{j}(P)}{f(P)}\right)^{n_{j}}\right\},
\end{aligned}
$$

where $h(n, P)=h\left(n, z_{1}, z_{2}, t\right)$ is an arbitrary function of the form

$$
h(n, P)=\sum_{0 \leq j \leq k,|\alpha|=k} h_{j \alpha}(n) z_{1}^{j} z_{2}^{k-j} t^{\alpha},
$$

$\alpha=\left(\alpha_{1}, \ldots, \alpha_{g}\right), t^{\alpha}=t_{1}^{\alpha_{1}} \cdots \cdot t_{g}^{\alpha_{g}}$, and satisfies the equation

$$
h\left(n, a_{1}, b_{1}, t\right)-\Lambda A^{k} h\left(n, a_{2}, b_{2}, \mathcal{P}(t)\right) \prod_{j=1}^{g}\left(\frac{A}{c_{j}}\right)^{n_{j}}=0 .
$$


This equation is equivalent to a set of linear homogeneous equations for $\left\{h_{j \alpha}(n)\right\}$ which has nontrivial solutions under the generic conditions in Proposition 1 [12. Notice that (13) can be written as

$$
\psi\left(n, a_{1}, b_{1}, t\right)-\Lambda \psi\left(n, a_{2}, b_{2}, \mathcal{P}(t)\right)=0 .
$$

According to (8), (9), (14), if $\psi \in \hat{M}_{\Lambda}(k)$, then $T_{j} \psi=\psi\left(n+e_{i}, P\right) \in \hat{M}_{\Lambda}(k+1)$. Consequently, we have $g$ mappings

$$
T_{j}: \hat{M}_{\Lambda}(k) \rightarrow \hat{M}_{\Lambda}(k+1), j=1, \ldots, g .
$$

Theorem 2. For an uncountable number of $h \in\left(\mathbb{C}^{*}\right)^{g}$ the module $\hat{M}_{\Lambda}$ is a free $\mathcal{T}_{g}$-module of rank $g$ generated by $g$ functions from $\hat{M}_{\Lambda}(1)$.

Let $A$ be the ring of meromorphic functions on $\Gamma$ with poles only on the divisor $(f=0)$.

Corollary 2. For values of $h$ specified in Theorem 2 there is an embedding of the ring

$$
A \rightarrow \operatorname{Mat}\left(g, \mathcal{T}_{g}\right) .
$$

In the case $g=2$ there is another way of identification of two lines on $\mathbb{C} P^{1} \times \mathbb{C} P^{1}$ which is suitable for our goals.

We set

$$
\Omega=\mathbb{C} P^{1} \times \mathbb{C} P^{1} /\left\{\left([1,0],\left[t_{1}, t_{2}\right]\right) \sim\left(\left[t_{1}, t_{2}\right],[0,1]\right)\right\} .
$$

Let $G, G_{1}, G_{2}$ be the functions on $\mathbb{C}^{4}$ of the form:

$$
\begin{gathered}
G\left(z_{1}, z_{2}, w_{1}, w_{2}\right)=\alpha_{0} z_{1} w_{1}+\beta_{0} z_{1} w_{2}+\gamma_{0} z_{2} w_{1}+\delta_{0} z_{2} w_{2}, \alpha, \beta, \gamma, \delta \in \mathbb{C}, \\
G_{i}\left(z_{1}, z_{2}, w_{1}, w_{2}\right)=\alpha_{i} z_{1} w_{1}+\beta_{i} z_{1} w_{2}+\gamma_{i} z_{2} w_{1}+\delta_{i} z_{2} w_{2}, \alpha_{i}, \beta_{i}, \gamma_{i}, \delta_{i} \in \mathbb{C} .
\end{gathered}
$$

Proposition 2 ([12]). For generic $\left(\alpha_{i}, \beta_{i}, \gamma_{i}, \delta_{i}\right) \in \mathbb{C}^{4}, i=0,1,2$, there exist $B, c_{1}, c_{2} \in$ $\mathbb{C}^{*}$ such that for every $t=\left(t_{1}, t_{2}\right) \in \mathbb{C}^{2}$ the functions $G, G_{1}, G_{2}$ satisfy the following equations:

$$
\begin{aligned}
G(0,1,0,1) & \neq 0, \\
G\left(1,0, t_{1}, t_{2}\right)-B G\left(t_{1}, t_{2}, 0,1\right) & =0 \\
G_{i}\left(1,0, t_{1}, t_{2}\right)-c_{i} G_{i}\left(t_{1}, t_{2}, 0,1\right) & =0 \quad i=1,2 .
\end{aligned}
$$

For any $\Lambda \in \mathbb{C}^{*}$ the discrete Baker-Akhiezer module $\hat{M}_{\Omega, \Lambda}=\bigcup_{k=0}^{\infty} \hat{M}_{\Omega, \Lambda}(k)$ in this case is defined by

$$
\hat{M}_{\Omega, \Lambda}(k)=\left\{\varphi=\frac{\tilde{h}\left(n_{1}, n_{2}, P\right)}{G(P)^{k}} \prod_{j=1}^{2}\left(\frac{G_{j}(P)}{G(P)}\right)^{n_{j}}\right\},
$$

where $\tilde{h}$ is an arbitrary function of the form (12) and satisfies

$$
\tilde{h}\left(n_{1}, n_{2}, 1,0, t_{1}, t_{2}\right)-\Lambda B^{k} \tilde{h}\left(n_{1}, n_{2}, t_{1}, t_{2}, 0,1\right) \prod_{j=1}^{2}\left(\frac{B}{c_{j}}\right)^{n_{j}}=0 .
$$

This equation is equivalent to a set of linear homogeneous equations for $\left\{h_{j \alpha}\right\}$ which has nontrivial solutions under the generic conditions in Proposition 2 [12]. Notice that (19) can be written as

$$
\varphi\left(n_{1}, n_{2}, 1,0, t_{1}, t_{2}\right)-\Lambda \varphi\left(n_{1}, n_{2}, t_{1}, t_{2}, 0,1\right)=0 .
$$

Theorem 3. For an uncountable number of $h \in\left(\mathbb{C}^{*}\right)^{2}$ the module $\hat{M}_{\Omega, \Lambda}$ is a free $\mathcal{T}_{2}$ module of rank 2 generated by two functions from $\hat{M}_{\Omega, \Lambda}(1)$. 
Let $A$ denote the ring of the meromorphic functions on $\Omega$ with poles only on the curve defined by the equation $G(P)=0$.

Corollary 3. For values of $h$ specified in Theorem 3 there is a ring embedding,

$$
A \rightarrow \operatorname{Mat}\left(2, \mathcal{T}_{2}\right)
$$

\section{Proofs}

Theorems 1 to 3 follow from their differential analogues. Since the schemes of the proofs are similar, we only prove Theorem 1 and Theorem 2 .

\subsection{Proof of Theorem 1, Let}

$$
\nabla_{i}=\partial_{i}-\zeta_{i}(z), \quad \partial_{i}=\partial / \partial x_{i}
$$

It is easy to see that it acts on $M_{c}$. Let $\mathcal{D}=\mathcal{K}\left[\partial_{1}, \ldots, \partial_{g}\right]$. Then $M_{c}$ is a $\mathcal{D}$-module. It is called the Baker-Akhiezer module of $(X, \Theta)$ [8]. Let

$$
\operatorname{gr} M_{c}=\oplus_{i} \operatorname{gr}_{i} M_{c}, \quad \operatorname{gr}_{i} M_{c}=M_{c}(i) / M_{c}(i-1) .
$$

Since $\partial_{i} M_{c}(m) \subset M_{c}(m+1), \operatorname{gr} M_{c}$ is also a $\mathcal{D}$-module. Recall that we assume that $\Theta$ is nonsingular in this paper. Then the following theorem is proved in [8].

Theorem 4. The module gr $M_{c}$ is a free $\mathcal{D}$-module of rank $g$ !.

More precisely, there exists a $\mathcal{D}$-free basis $\varphi_{i j}$ such that $\varphi_{i j} \in \operatorname{gr}_{i} M_{c}, 1 \leq i \leq g$, $1 \leq j \leq r_{j}$ with

$$
r_{i}=i^{g}-(i-1)^{g}-\sum_{j=1}^{i-1} r_{j}\left(\begin{array}{c}
g+i-j-1 \\
g-1
\end{array}\right), \quad r \geq 2,
$$

and $r_{1}=1$. Moreover, for each $i$, one can find $\varphi_{i j}$ in $\left\{F_{i, a}(z, x)\right\}$; that is, one can write

for some $a_{i j} \in \mathbb{Z}^{g} / i \mathbb{Z}^{g}$.

$$
\varphi_{i j}=F_{i, a_{i j}}(z, x)
$$

We remark that, in Theorem 1] $c=0$ is not excluded. This is because we consider $\mathcal{K}$, the space of meromorphic functions of $x$, as a coefficient field of $\mathcal{D}$ and $M_{c}$.

Recall that $T_{i}$ acts also on $M_{c}$. It satisfies

$$
T_{i} M_{c}(m) \subset M_{c}(m+1) .
$$

Therefore $T_{i}$ acts on $\operatorname{gr} M_{c}$ too. For $F(z, x) \in M_{c}$ we have the expansion

$$
T_{i} F(z, x)=F(z, x)+\nabla_{i} F(z, x) h_{i}+O\left(h_{i}^{2}\right),
$$

and it is possible to define the map $\tilde{T}_{i}=\left(T_{i}-1\right) / h_{i}: M_{c} \rightarrow M_{c}$ :

$$
\tilde{T}_{i} F(z, x)=\frac{1}{h_{i}}\left(T_{i} F(z, x)-F(z, x)\right) .
$$

It satisfies

$$
\tilde{T}_{i} F(z, x)=\nabla_{i} F(z, x)+O\left(h_{i}\right) .
$$

Notice that, as an action on $\operatorname{gr} M_{c}$,

$$
\tilde{T}_{i}=\frac{1}{h_{i}} T_{i}
$$

We prove

Theorem 5. For an uncountable number of $h \in\left(\mathbb{C}^{*}\right)^{g}, \operatorname{gr} M_{c}$ is a free $\mathcal{T}_{g}$-module of rank $g$ ! with a basis $\left\{\varphi_{i j}\right\}$. 
Proof. Since gr $M_{c}$ is a free $\mathcal{D}$ module, for each $k$, the set of elements

$$
\begin{aligned}
& \partial_{1}^{k_{1}} \cdots \partial_{g}^{k_{g}} \varphi_{i j}, \\
& k_{1}+\cdots+k_{g}=k^{\prime}-i, \quad 0 \leq k^{\prime} \leq k, \quad 1 \leq i \leq g, \quad 1 \leq j \leq r_{i},
\end{aligned}
$$

is a $\mathcal{K}$-basis of $M_{c}(k)$. The number of elements in (21) is $N_{k}:=k^{g}$. Let us enumerate them as $\psi_{1}^{k}, \ldots, \psi_{N_{k}}^{k}$.

Expand

$$
\theta(z)^{k} \psi_{i}^{k}=\sum a_{i, \mu}^{k}(x) z^{\mu}, \quad \mu=\left(\mu_{1}, \ldots, \mu_{g}\right) .
$$

Since $\left\{\psi_{i}^{k}\right\}$ is linearly independent over $\mathcal{K}$, there exist $\mu^{(k, 1)}, \ldots, \mu^{\left(k, N_{k}\right)}$ such that

$$
\operatorname{det}\left(a_{i, \mu^{(k, j)}}^{k}(x)\right)_{1 \leq i, j \leq N_{k}} \neq 0,
$$

where " $\neq 0$ " signifies that it is not identically zero as a function of $x$.

Consider correspondingly that

$$
\tilde{T}_{1}^{k_{1}} \cdots \tilde{T}_{g}^{k_{g}} \varphi_{i j}
$$

Let us denote the function in (22) which has the same $\left(k_{1}, \ldots, k_{g}\right)$ as $\psi_{i}^{k}$ by $\tilde{\psi}_{i}^{k}$. Then

$$
\tilde{\psi}_{i}^{k}(z, x, h)=\psi_{i}^{k}(z, x)+\sum_{l=1}^{g} O\left(h_{l}\right) .
$$

If we expand

$$
\theta(z)^{k} \tilde{\psi}_{i}^{k}=\sum \tilde{a}_{i, \mu}^{k}(x, h) z^{\mu}
$$

then

$$
\tilde{a}_{i, \mu}^{k}(x, h)=a_{i, \mu}^{k}(x)+\sum_{l=1}^{g} O\left(h_{l}\right)
$$

and

$$
\operatorname{det}\left(\tilde{a}_{i, \mu^{(k, j)}}^{k}(x, 0)\right)=\operatorname{det}\left(a_{i, \mu^{(j)}}^{k}(x)\right) \neq 0 .
$$

Notice that $\operatorname{det}\left(a_{i, \mu^{(k, j)}}^{k}(x)\right)$ is an analytic function of $x$ and the zero set of it is of measure zero. Thus

$$
\mathbb{C}^{g} \backslash \bigcup_{k=0}^{\infty}\left(\operatorname{det}\left(a_{i, \mu^{(k, j)}}^{k}(x)\right)=0\right)
$$

has positive measure and contains an uncountable number of elements. Take any $x_{0}$ from (23). Since $\tilde{a}_{i, \mu}^{k}(x, h)$ is an analytic function of $(x, h)$, the set

$$
\mathbb{C}^{g} \backslash \bigcup_{k=0}^{\infty}\left\{h \mid \operatorname{det}\left(\tilde{a}_{i, \mu^{(k, j)}}^{k}\left(x_{0}, h\right)\right)=0\right\}
$$

contains an uncountable number of elements. Moreover, it contains elements of the form $h_{0}=\left(h_{01}, \ldots, h_{0 g}\right), h_{0 i} \neq 0$, for any $i$, since $\bigcup_{i=1}^{g}\left\{\sum_{j \neq i} h_{j} e_{j} \in \mathbb{C}^{g} \mid h_{j} \in \mathbb{C}^{g}\right\}$ is also of measure zero. Take such an $h_{0}$. Then, for any $k$,

$$
\operatorname{det}\left(\tilde{a}_{i, \mu^{(k, j)}}^{k}\left(x, h_{0}\right)\right) \neq 0 \text {. }
$$

For such an $h_{0}\left\{\tilde{\psi}_{i}^{k}\right\}$ is linearly independent and generates $M_{c}(k)$ over $\mathcal{K}$ for all $k \geq 0$. Therefore gr $M_{c}$ is a free $\mathcal{T}_{g}$ module with the basis $\left\{\varphi_{i j}\right\}$. 
Let us prove Theorem 1. Notice that $T_{i}$ satisfies the following commutation relation with a function of $x$ :

$$
T_{i} F(x)=F\left(x+h_{i} e_{i}\right) T_{i} .
$$

By definition the discretization $\hat{\varphi}_{i j}(n)$ of $\varphi_{i j}(z, x)$ is

$$
\hat{\varphi}(n)=T^{n} \varphi_{i j}(z, x) .
$$

By Theorem 5 any element of $M_{c}$ can be uniquely written as a linear combination of

$$
T^{m} \varphi_{i j}, \quad m \in \mathbb{Z}_{\geq 0}^{g}, \quad 1 \leq i \leq g, \quad 1 \leq j \leq r_{i},
$$

with the coefficients in $\mathcal{K}$. The discretization of the element of the form $f(x) T^{m} \varphi_{i j}$ with $f(x) \in \mathcal{K}$ is given by

$$
T^{n}\left(f(x) T^{m} \varphi_{i j}(z, x)\right)=f(x+n h) T^{m} T^{n} \varphi_{i j}(z, x)=\hat{f}(n) T^{m} \hat{\varphi}_{i j}(n) .
$$

Thus any element of $\hat{M}_{c}$ can be written as a linear combination of $\left\{T^{m} \hat{\varphi}_{i j}(n)\right\}$ with the coefficients in $\hat{\mathcal{K}}$.

Moreover, this description of an element of $\hat{M}_{c}$ as a linear combination of $\left\{T^{m} \hat{\varphi}_{i j}(n)\right\}$ is unique. In fact, suppose that

$$
\sum \hat{f}_{i j}(n) T^{m_{i j}} \hat{\varphi}_{i j}(n)=0
$$

Applying $T^{-n}$ to (25) we get

$$
\sum f_{i j}(x) T^{m_{i j}} \varphi_{i j}(z, x)=0
$$

It follows that $f_{i j}(x)=0$ for any $(i, j)$, since $\left\{\varphi_{i j}(z, x)\right\}$ is linearly independent over $\mathcal{K}$. Consequently $\hat{f}_{i j}(n)=0$ for every $(i, j)$.

Thus $\hat{M}_{c}$ is proved to be a free $\mathcal{T}_{g}$-module with a basis $\hat{\varphi}_{i j}$.

3.2. Proof of Theorem 2, We shall first give a construction of functions $f, f_{i}$ satisfying conditions (5)-(9) together with further conditions and related functions $\tilde{f}_{i}$.

Consider the function $F\left(z_{1}, z_{2}, t, s\right), t \in \mathbb{C}^{g}, s \in \mathbb{C}$, of the form

$$
F\left(z_{1}, z_{2}, t, s\right)=\sum_{k=1}^{g}\left(\gamma_{k}(s) z_{1}+\delta_{k}(s) z_{2}\right) t_{k}
$$

and the following equation for $F$ :

$$
F\left(a_{1}, b_{1}, t, s\right)=A e^{s} F\left(a_{2}, b_{2}, \mathcal{P}(t), s\right) .
$$

This equation gives $g$ linear homogeneous equations for $2 g$ unknown variables $\gamma_{k}, \delta_{k}$. By examining the case $\left(a_{1}, b_{1}\right)=(1,0),\left(a_{2}, b_{2}\right)=(0,1), \mathcal{P}(t)=\left(\lambda_{1} t_{1}, \ldots, \lambda_{g} t_{g}\right)$, we see easily that, for generic choice of $a_{i}, b_{i}, \mathcal{P}$, there exist $g$ linearly independent solutions $\left\{F_{i}\right\}$ of (26) such that the following conditions are satisfied:

(i) $F_{i}\left(z_{1}, z_{2}, t, 0\right)$ is independent of $i$. Set $f\left(z_{1}, z_{2}, t\right)=F_{i}\left(z_{1}, z_{2}, t, 0\right)$.

(ii) The set of functions $\left\{f, \partial_{s} F_{i}\left(z_{1}, z_{2}, t, 0\right)\right\}$ is linearly independent.

(iii) $f\left(a_{1}, b_{1}, v_{j}\right) \neq 0$ for any $j$.

We take $\tilde{c}_{i}, h_{i} \in \mathbb{C}^{*}$ and set

$$
c_{i}=A e^{\tilde{c}_{i} h_{i}} .
$$

We define $f_{i}$ and $\tilde{f}_{i}$ by

$$
\begin{aligned}
f_{i}\left(z_{1}, z_{2}, t, h_{i}\right) & =F_{i}\left(z_{1}, z_{2}, t, \tilde{c}_{i} h_{i}\right), \\
\tilde{f}_{i}\left(z_{1}, z_{2}, t\right) & =\tilde{c}_{i} \partial_{s} F_{i}\left(z_{1}, z_{2}, t, 0\right) .
\end{aligned}
$$


Then $f, f_{i}$ satisfy (5)-(9) and $\tilde{f}_{i}$ satisfies

$$
\begin{aligned}
& \tilde{f}_{i}\left(z_{1}, z_{2}, t\right)=\partial_{h_{i}} f_{i}\left(z_{1}, z_{2}, t, 0\right), \\
& \tilde{f}_{i}\left(a_{1}, b_{1}, t\right)-A \tilde{f}_{i}\left(a_{2}, b_{2}, \mathcal{P}(t)\right)-\tilde{c}_{i} f\left(a_{1}, b_{1}, t\right)=0,
\end{aligned}
$$

due to (26). Moreover, by property (ii), $\left\{f, \tilde{f}_{1}, \ldots, \tilde{f}_{g}\right\}$ is linearly independent.

Next we consider, for $k$ fixed, a function $h(x, P), P \in \mathbb{C}^{g+2}$, such that

$$
\begin{aligned}
& h(x, P)=\sum_{0 \leq j \leq k,|\alpha|=k} h_{j \alpha}(x) z_{1}^{j} z_{2}^{k-j} t^{\alpha}, \\
& \frac{h\left(x, a_{1}, b_{1}, t\right)}{f\left(a_{1}, b_{1}, t\right)^{k}}-\Lambda e^{-\sum_{i=1}^{g} \tilde{c}_{i} x_{i}} \frac{h\left(x, a_{2}, b_{2}, \mathcal{P}(t)\right)}{f\left(a_{2}, b_{2}, \mathcal{P}(t)\right)^{k}}=0 .
\end{aligned}
$$

Equation (32) is equivalent to the system of linear homogeneous equations for $\left\{h_{j \alpha}\right\}$. As shown in [12,

$$
\sharp\left\{h_{j \alpha}\right\}-\sharp\{\text { equations }\}=g\left(\begin{array}{c}
g+k-1 \\
g
\end{array}\right),
$$

where $\sharp S$ denotes the number of elements of $S$. Therefore (32) has non-trivial solutions. Moreover, it is possible take a basis of solutions such that each element of a basis is a rational function of $e^{\sum_{i=1}^{g} \tilde{c}_{i} x_{i}}$ and is analytic at $x=0$.

Let $\mathcal{K}$ be the ring of rational functions of $e^{\sum_{i=1}^{g} \tilde{c}_{i} x_{i}}$ and

$$
M_{\lambda}=\bigcup_{k=0}^{\infty} M_{\lambda}(k), \quad M_{\Lambda}(k)=\left\{\frac{h(x, P)}{f(P)^{k}}\right\},
$$

where $h(x, P)$ is an arbitrary rational function of $e^{\sum_{i=1}^{g} \tilde{c}_{i} x_{i}}$ that satisfies (31), (32). Obviously $M_{\Lambda}$ is a vector space over $\mathcal{K}$. As remarked above we can take a basis of each $M_{\Lambda}(k)$ over $\mathcal{K}$ such that each element of the basis is analytic at $x=0$.

Equation (32) signifies that an element $\varphi(x, P)$ of $M_{\Lambda}$ satisfies

$$
\varphi\left(x, a_{1}, b_{1}, t\right)-\Lambda e^{-\sum_{i=1}^{g} \tilde{c}_{i} x_{i}} \varphi\left(x, a_{2}, b_{2}, \mathcal{P}(t)\right)=0 .
$$

Let

$$
\xi_{i}(P)=\frac{\tilde{f}_{i}(P)}{f_{i}(P)}
$$

Then it satisfies that

$$
\xi_{i}\left(a_{1}, b_{1}, t\right)-\xi_{i}\left(a_{2}, b_{2}, \mathcal{P}(t)\right)-\tilde{c}_{i}=0
$$

due to (30). We set

$$
\nabla_{i}=\partial_{i}+\xi_{i}(P) .
$$

Using (33) one can easily check that $\nabla_{i}$ acts on $M_{\Lambda}$ and satisfies

$$
\nabla_{i} M_{\Lambda}(k) \subset M_{\Lambda}(k+1) .
$$

Thus $M_{\Lambda}$ and gr $M_{\Lambda}$ become modules over the ring of differential operators $\mathcal{D}:=\mathcal{K}\left[\partial_{1}, \ldots, \partial_{g}\right]$, where $\partial_{i}$ acts by $\nabla_{i}$. The $\mathcal{D}$-module $M_{\Lambda}$ is the Baker-Akhiezer module of $(\Gamma,(f=0))$ constructed in 12 .

The following theorem had been proved in 12 .

Theorem 6. The module gr $M_{\Lambda}$ is a free $\mathcal{D}$-module of rank g generated by $g$ functions from $M_{\Lambda}(1)$. 
Similarly to the case of abelian varieties we define the operator $T_{i}$ by

$$
T_{i}=\frac{f_{i}\left(P, h_{i}\right)}{f(P)} \mathrm{e}^{h_{i} \partial_{i}}
$$

where $\mathrm{e}^{h_{i} \partial_{i}}$ is the shift operator:

$$
\mathrm{e}^{h_{i} \partial_{i}} G\left(\ldots, x_{i}, \ldots\right)=G\left(\ldots, x_{i}+h_{i}, \ldots\right) .
$$

By (8) and (9) $T_{i}$ acts on $M_{\Lambda}$ and satisfies $T_{i} M_{\Lambda}(k) \subset M_{\Lambda}(k+1)$. Therefore $T_{i}$ acts on $\operatorname{gr} M_{\Lambda}$.

By (29) we have

$$
f_{i}\left(P, h_{i}\right)=f(P)+\tilde{f}_{i}(P) h_{i}+O\left(h_{i}^{2}\right)
$$

Consequently,

$$
T_{i}=1+h_{i} \nabla_{i}+O\left(h_{i}^{2}\right)
$$

We set

$$
\tilde{T}_{i}=\frac{1}{h_{i}}\left(T_{i}-1\right)=\nabla_{i}+O\left(h_{i}\right)
$$

On $\operatorname{gr} M_{\Lambda}$ we have

$$
\tilde{T}_{i}=\frac{1}{h_{i}} T_{i}
$$

The discretization $\hat{M}_{\Lambda}$ of $M_{\Lambda}$ is defined similarly to the case of Abelian varieties using $T_{i}$. Explicitly, $\hat{M}_{\Lambda}$ is given by (10) and (111).

The proof of Theorem 2 is completely similar to that of Theorem 1 and reduces to Theorem [ ] using $\tilde{T}_{i}$.

\section{Commuting Difference operators}

In this section we give examples of explicit forms of commuting difference operators.

4.1. Two-point operators: $g=1$. Let $g=1$ in Theorem $1, X=\mathbb{C} /(\mathbb{Z}+\tau \mathbb{Z})$. In this case the DBA module $\hat{M}_{0}$ is generated over $\mathcal{T}_{1}$ by the function

$$
\psi(n, z)=\frac{\theta(z+x+n h)}{\theta(z)}\left(\frac{\theta(z-h)}{\theta(z)}\right)^{n} \in \hat{M}_{c}(1) .
$$

Let

$$
\lambda=\frac{\theta(z-h) \theta(z+h)}{\theta^{2}(z)} .
$$

There is a unique operator of the form

$$
L_{1}=v_{2}(n) T^{2}+v_{1}(n) T+v_{0}(n)
$$

such that

$$
L_{1} \psi(n, z)=\lambda(z) \psi(n, z) .
$$

Let us find the coefficients $v_{i}(n)$. We divide (34) by $(\theta(z-h) / \theta(z))^{n}$ and multiply by $\theta(z)^{3}$ :

$$
\begin{aligned}
& v_{2}(n) \theta(z+x+(n+2) h) \theta^{2}(z-h)+v_{1}(n) \theta(z+x+(n+1) h) \theta(z-h) \theta(z) \\
& +v_{0}(n) \theta(z+x+n h) \theta(z)^{2}=\theta(z-h) \theta(z+h) \theta(z+x+n h) .
\end{aligned}
$$


We recall that $\theta\left(\frac{1}{2}+\frac{1}{2} \tau\right)=0$. Let us substitute $z=p=\frac{1}{2}+\frac{1}{2} \tau+h$ in (35). We obtain $v_{0}=0$. Let us divide (35) by $\theta(z-h)$ and again substitute $z=p=\frac{1}{2}+\frac{1}{2} \tau+h$. We obtain

$$
v_{1}(n)=\frac{\theta(p+x+n h) \theta(p+h)}{\theta(p+x+(n+1) h) \theta(p)} .
$$

We put $z=q=\frac{1}{2}+\frac{1}{2} \tau$ in (35) and obtain

$$
v_{2}(n)=\frac{\theta(q+x+n h) \theta(q+h)}{\theta(q+x+(n+2) h) \theta(q-h)} .
$$

Similarly, for

$$
\mu=\frac{\theta(z-h) \theta^{2}\left(z+\frac{h}{2}\right)}{\theta^{3}(z)}
$$

we have

$$
L_{2} \psi=\left(u_{3}(n) T^{3}+u_{2}(n) T^{2}+u_{1}(n) T+u_{0}(n)\right) \psi=\mu \psi .
$$

From (36) we obtain $u_{0}=0$,

$$
u_{1}(n)=\frac{\theta(p+x+n h) \theta^{2}\left(p+\frac{h}{2}\right)}{\theta(p+x+(n+1) h) \theta^{2}(p)}, \quad u_{3}(n)=\frac{\theta(q+x+n h) \theta^{2}\left(q+\frac{h}{2}\right)}{\theta(q+x+(n+3) h) \theta^{2}(q-h)} .
$$

To find $u_{2}(n)$ let us substitute $z=r=\frac{1}{2}+\frac{1}{2} \tau-\frac{h}{2}$ in (36):

$$
u_{2}(n)=-u_{1}(n) \frac{\theta(r+x+(n+1) h) \theta(r)}{\theta(r+x+(n+2) h) \theta(r-h)}-u_{3}(n) \frac{\theta(r+x+(n+3) h) \theta(r-h)}{\theta(r+x+(n+2) h) \theta(r)} .
$$

Operators $L_{1}$ and $L_{2}$ commute.

It is easy to see that for the meromorphic function $\eta$ with poles at $p$ and $q$ there is an operator of the form

$$
L=\sum_{i=N_{-}}^{N_{+}} v_{i}(n) T^{i}
$$

such that

$$
L \psi=\eta \psi .
$$

We see that in the case $g=1$ our construction is involved in the two-points construction [2].

4.2. $2 \times 2$-matrix operators: Abelian varieties. Let $g=2$ in Theorem $1, X=$ $\mathbb{C}^{2} /\left(\mathbb{Z}^{2}+\tau \mathbb{Z}^{2}\right)$. The functions

$$
\begin{aligned}
& \psi_{1}=\frac{\theta(z+x+n h)}{\theta(z)} \prod_{j=1}^{2}\left(\frac{\theta\left(z-h_{j} e_{j}\right)}{\theta(z)}\right)^{n_{j}} \in \hat{M}_{0}(1), \\
& \psi_{2}=\frac{\theta(z+x+n h+\beta) \theta(z-\beta)}{\theta^{2}(z)} \prod_{j=1}^{2}\left(\frac{\theta\left(z-h_{j} e_{j}\right)}{\theta(z)}\right)^{n_{j}} \in \hat{M}_{0}(2)
\end{aligned}
$$

form a basis in $\hat{M}_{0}$, where $\beta$ belongs to some open everywhere dense subset in $\mathbb{C}^{2}$. Let us find the operator corresponding to the function

$$
\lambda=\frac{\theta\left(z-h_{1} e_{1}\right) \theta\left(z+h_{1} e_{1}\right)}{\theta^{2}(z)} .
$$

We have

$$
\begin{aligned}
& L_{11} \psi_{1}+L_{12} \psi_{2}=\lambda \psi_{1}, \\
& L_{21} \psi_{1}+L_{22} \psi_{2}=\lambda \psi_{2} .
\end{aligned}
$$


Operators $L_{11}$ and $L_{12}$ have the form

$$
L_{11}=v_{20} T_{1}^{2}+v_{11} T_{1} T_{2}+v_{02} T_{2}^{2}+v_{1} T_{1}+v_{2} T_{2}+v_{0}, \quad L_{12}=u_{1} T_{1}+u_{2} T_{2}+u_{0} .
$$

Let us divide (38) by $\prod_{j=1}^{2}\left(\theta\left(z-h_{j} e_{j}\right) / \theta(z)\right)^{n_{j}}$ and multiply by $\theta(z)^{3}$. Then we get

$$
\begin{aligned}
& v_{20} \theta\left(z+x+n h+2 h_{1} e_{1}\right) \theta\left(z-h_{1} e_{1}\right)^{2} \\
& +v_{11} \theta(z+x+(n+1) h) \theta\left(z-h_{1} e_{1}\right) \theta\left(z-h_{2} e_{2}\right) \\
& +v_{02} \theta\left(z+x+n h+2 h_{2} e_{2}\right) \theta\left(z-h_{2} e_{2}\right)^{2} \\
& +v_{1} \theta\left(z+x+n h+h_{1} e_{1}\right) \theta\left(z-h_{1} e_{1}\right) \theta(z) \\
& +v_{2} \theta\left(z+x+n h+h_{2} e_{2}\right) \theta\left(z-h_{2} e_{2}\right) \theta(z)+v_{0} \theta(z+x+n h) \theta(z)^{2} \\
& +u_{1} \theta\left(z+x+n h+h_{1} e_{1}+\beta\right) \theta(z-\beta) \theta\left(z-h_{1} e_{1}\right) \\
& +u_{2} \theta\left(z+x+n h+h_{2} e_{2}+\beta\right) \theta(z-\beta) \theta\left(z-h_{2} e_{2}\right) \\
& +u_{0} \theta(z+x+n h+\beta) \theta(z-\beta) \theta(z)=\theta\left(z-h_{1} e_{1}\right) \theta\left(z+h_{1} e_{1}\right) \theta(z+x+n h) .
\end{aligned}
$$

\section{Lemma 1. The equalities}

$$
v_{0}=u_{0}=0
$$

are valid.

Proof. Let $p_{1}$ and $p_{2}$ be the points of intersection of the curves $\theta\left(z-h_{1} e_{1}\right)=0$ and $\theta\left(z-h_{2} e_{2}\right)=0$. Let us substitute $z=p_{1}$ and $z=p_{2}$ in (39):

$$
v_{0} \theta\left(p_{i}+x+n h\right) \theta\left(p_{i}\right)^{2}+u_{0} \theta\left(p_{i}+x+n h+\beta\right) \theta\left(p_{i}-\beta\right) \theta\left(p_{i}\right)=0 .
$$

These equations can be considered as a system of linear equations for $v_{0}, u_{0}$. If $v_{0} \neq 0$ or $u_{0} \neq 0$, then

$$
\begin{aligned}
& \theta\left(p_{1}+x+n h\right) \theta\left(p_{2}+x+n h+\beta\right) \theta\left(p_{2}-\beta\right) \theta\left(p_{1}\right) \\
& -\theta\left(p_{2}+x+n h\right) \theta\left(p_{1}+x+n h+\beta\right) \theta\left(p_{1}-\beta\right) \theta\left(p_{2}\right)=0 .
\end{aligned}
$$

If $\beta$ is a solution of $\theta\left(p_{1}-\beta\right)=0$, then this equality is not valid. Consequently, for $\beta$ in general position this equality is not valid. Thus Lemma 1 is proved.

Let us restrict (39) on the curve $\theta\left(z-h_{1} e_{1}\right)=0$ and divide by $\theta\left(z-h_{2} e_{2}\right)$ :

$$
\begin{gathered}
v_{02} \theta\left(z+x+n h+2 h_{2} e_{2}\right) \theta\left(z-h_{2} e_{2}\right)+v_{2} \theta\left(z+x+n h+h_{2} e_{2}\right) \theta(z) \\
+u_{2} \theta\left(z+x+n h+h_{2} e_{2}+\beta\right) \theta(z-\beta)=0 .
\end{gathered}
$$

Let $q_{1}$ and $q_{2}$ be the points of intersection of $\theta\left(z-h_{1} e_{1}\right)=0$ and $\theta(z)=0$. Then

$$
v_{02} \theta\left(q_{i}+x+n h+2 h_{2} e_{2}\right) \theta\left(q_{i}-h_{2} e_{2}\right)+u_{2} \theta\left(q_{i}+x+n h+h_{2} e_{2}+\beta\right) \theta\left(q_{i}-\beta\right)=0 .
$$

By a similar argument as in the proof of Lemma 1 we obtain

$$
v_{02}=v_{2}=u_{2}=0 \text {. }
$$

We divide (39) by $\theta\left(z-h_{1} e_{1}\right)$ and get

$$
\begin{gathered}
v_{20} \theta\left(z+x+n h+2 h_{1} e_{1}\right) \theta\left(z-h_{1} e_{1}\right)+v_{11} \theta(z+x+(n+1) h) \theta\left(z-h_{2} e_{2}\right) \\
+v_{1} \theta\left(z+x+n h+h_{1} e_{1}\right) \theta(z)+u_{1} \theta\left(z+x+n h+h_{1} e_{1}+\beta\right) \theta(z-\beta) \\
=\theta(z+x+n h) \theta\left(z+h_{1} e_{1}\right) .
\end{gathered}
$$

Let us substitute $z=p_{1}$ and $z=p_{2}$ in (41). Then we obtain

$$
\left(\begin{array}{l}
v_{1} \\
u_{1}
\end{array}\right)=A_{1}^{-1}\left(\begin{array}{c}
\theta\left(p_{1}+x+n h\right) \theta\left(p_{1}+h_{1} e_{1}\right) \\
\theta\left(p_{2}+x+n h\right) \theta\left(p_{2}+h_{1} e_{1}\right)
\end{array}\right)
$$




$$
A_{1}=\left(\begin{array}{cc}
\theta\left(p_{1}+x+n h+h_{1} e_{1}\right) \theta\left(p_{1}\right) & \theta\left(p_{1}+x+n h+h_{1} e_{1}+\beta\right) \theta\left(p_{1}-\beta\right) \\
\theta\left(p_{2}+x+n h+h_{1} e_{1}\right) \theta\left(p_{2}\right) & \theta\left(p_{2}+x+n h+h_{1} e_{1}+\beta\right) \theta\left(p_{2}-\beta\right)
\end{array}\right) .
$$

Let $r_{1}$ and $r_{2}$ be the points of intersection of $\theta(z)=0$ and $\theta(z-\beta)=0$. From (41) we obtain

$$
\begin{gathered}
\left(\begin{array}{c}
v_{20} \\
v_{11}
\end{array}\right)=A_{2}^{-1}\left(\begin{array}{c}
\theta\left(r_{1}+x+n h\right) \theta\left(r_{1}+h_{1} e_{1}\right) \\
\theta\left(r_{2}+x+n h\right) \theta\left(r_{2}+h_{1} e_{1}\right)
\end{array}\right), \\
A_{2}=\left(\begin{array}{cc}
\theta\left(r_{1}+x+n h+2 h_{1} e_{1}\right) \theta\left(r_{1}-h_{1} e_{1}\right) & \theta\left(r_{1}+x+(n+1) h\right) \theta\left(r_{1}-h_{2} e_{2}\right) \\
\theta\left(r_{2}+x+n h+2 h_{1} e_{1}\right) \theta\left(r_{2}-h_{1} e_{1}\right) & \theta\left(r_{2}+x+(n+1) h\right) \theta\left(r_{2}-h_{2} e_{2}\right)
\end{array}\right) .
\end{gathered}
$$

Similarly it is possible to find operators $L_{21}, L_{22}$ and an operator corresponding to

$$
\frac{\theta\left(z-h_{2} e_{2}\right) \theta\left(z+h_{2} e_{2}\right)}{\theta^{2}(z)} \text {. }
$$

4.3. $2 \times 2$-matrix operators with rational coefficients. It is a well known fact that the Lame identity

$$
\begin{gathered}
\left(\partial_{x}^{2}-2 \wp(x)\right) \psi(x, z)=\wp(z) \psi(x, z), \\
\psi(x, z)=\frac{\sigma(z+x)}{\sigma(x) \sigma(z)} e^{-x \zeta(z)},
\end{gathered}
$$

where $\sigma, \zeta, \wp$ are Weierstrass functions of the elliptic curve $w^{2}=4 y^{3}+\alpha_{1} y+\alpha_{0}$, takes the form

$$
\begin{gathered}
\left(\partial_{x}^{2}-\frac{2}{x^{2}}\right) \psi^{\vee}(x, z)=\frac{1}{z^{2}} \psi^{\vee}(x, z), \\
\psi^{\vee}(x, z)=\frac{z+x}{x z} e^{-\frac{x}{z}}
\end{gathered}
$$

under the degeneration $\alpha_{i} \rightarrow 0$. The Lame potential becomes the rational function $-\frac{2}{x^{2}}$. In this section we shall consider the spectral variety $X^{\vee}$ obtained from the Abelian variety $X=\mathbb{C}^{2} /\left(\mathbb{Z}^{2}+\tau \mathbb{Z}^{2}\right)$ by a similar degeneration. Elements of the corresponding DBA module are expressed in terms of elementary functions; coefficients of commuting difference operators are rational functions. To describe $X^{\vee}$ we recall Mumford's construction of the affine part of the Jacobian variety of a hyperelliptic curve $\Sigma$ of genus $g$ (see [13]):

$$
w^{2}=f(y)=4 y^{2 g+1}+\alpha_{2 g} y^{2 g}+\cdots+\alpha_{0} .
$$

Let us introduce the polynomials

$$
a(y)=\sum_{i=1}^{g} a_{2 i+1} y^{g-i}, b(y)=\sum_{i=0}^{g} b_{2 i} y^{g-i}, c(y)=\sum_{i=0}^{g+1} c_{2 i} y^{g+1-i},
$$

$b_{0}=1, c_{0}=4, a_{1}=0$. We shall consider the affine space $\mathbb{C}^{3 g+1}$ with the coordinates $\left(a_{2 i+1}, b_{2 i}, c_{2 i}\right)$. The affine part $J(\Sigma) \backslash \Theta$ is given in $\mathbb{C}^{3 g+1}$ by the following system of equations for $a_{2 i+1}, b_{2 i}, c_{2 i}$ :

$$
a^{2}(y)+b(y) c(y)=f(y)
$$

In the case $g=2$ we have the following equations:

$$
\begin{gathered}
\alpha_{0}-a_{5}^{2}-b_{4} c_{6}=0, \alpha_{1}-2 a_{3} a_{5}-b_{4} c_{4}-b_{2} c_{6}=0, \\
\alpha_{2}-a_{3}^{2}-b_{4} c_{2}-b_{2} c_{4}-c_{6}=0, \alpha_{3}-4 b_{4}-b_{2} c_{2}-c_{4}=0, \alpha_{4}-4 b_{2}-c_{2}=0 .
\end{gathered}
$$

We define the spectral variety $X^{\vee}$ by the conditions $\alpha_{i}=0$. From the last three equations one can find $c_{2}, c_{4}, c_{6}$, and substitute it in the first two equations. One gets that $X^{\vee}$ is isomorphic to the variety defined in $\mathbb{C}^{4}$ by the two equations

$$
b_{4}\left(a_{3}^{2}+4 b_{2}^{3}-8 b_{2} b_{4}\right)-a_{5}^{2}=0, \quad a_{3}^{2} b_{2}-2 a_{3} a_{5}+4\left(b_{2}^{4}-3 b_{2}^{2} b_{4}+b_{4}^{2}\right)=0 .
$$

Analytically this degeneration of the Jacobian variety is well described by using the sigma function of $X$. The sigma function is a certain modification of Riemann's theta 
function which was originally introduced by Klein [14, 15]. The important property for us now is that the sigma function $\sigma\left(z_{1}, z_{2}\right)$ becomes the Schur function

$$
\sigma^{\vee}=\frac{z_{1}^{3}}{3}-z_{2}
$$

under the limit $\alpha_{i} \rightarrow 0$ [16, 17]. The $a_{i}, b_{i}, c_{i}$ coordinates of the Jacobian can be described explicitly using the sigma function (see [13), and, consequently, those of the variety given by (42) are described by the Schur function.

One can replace Riemann's theta function by the sigma function in the description of the DBA module on $X$ as in the previous section. A free basis of the DBA module is given by

$$
\begin{aligned}
& \psi_{1}=\frac{\sigma(z+x+n h)}{\sigma(z)} \prod_{j=1}^{2}\left(\frac{\sigma\left(z-h_{j} e_{j}\right)}{\sigma(z)}\right)^{n_{j}} \in \hat{M}_{0}(1), \\
& \psi_{2}=\frac{\sigma(z+x+n h+\beta) \sigma(z-\beta)}{\sigma^{2}(z)} \prod_{j=1}^{2}\left(\frac{\sigma\left(z-h_{j} e_{j}\right)}{\sigma(z)}\right)^{n_{j}} \in \hat{M}_{0}(2) .
\end{aligned}
$$

Taking the limit $\alpha_{i} \rightarrow 0$ we get a new free DBA module on $X^{\vee}$ generated by the functions

$$
\begin{aligned}
& \psi_{1}^{\vee}=\frac{\sigma^{\vee}(z+x+n h)}{\sigma^{\vee}(z)} \prod_{j=1}^{2}\left(\frac{\sigma^{\vee}\left(z-h_{j} e_{j}\right)}{\sigma^{\vee}(z)}\right)^{n_{j}}, \\
& \psi_{2}^{\vee}=\frac{\sigma^{\vee}(z+x+n h+\beta) \sigma^{\vee}(z-\beta)}{\left(\sigma^{\vee}(z)\right)^{2}} \prod_{j=1}^{2}\left(\frac{\sigma^{\vee}\left(z-h_{j} e_{j}\right)}{\sigma^{\vee}(z)}\right)^{n_{j}} .
\end{aligned}
$$

Let $\Psi={ }^{t}\left(\psi_{1}, \psi_{2}\right)$ and $\Psi^{\vee}=\left(\psi_{1}^{\vee}, \psi_{2}^{\vee}\right)$. As a limit of the identity $L(\lambda) \Psi=\lambda \Psi$ we get $L^{\vee}\left(\lambda^{\vee}\right) \Psi^{\vee}=\lambda^{\vee} \Psi^{\vee}$, where $\lambda^{\vee}$ is the corresponding limit of $\lambda$. For different $\lambda^{\vee}$ and $\mu^{\vee}$ operators $L^{\vee}\left(\lambda^{\vee}\right)$ and $L^{\vee}\left(\mu^{\vee}\right)$ commute. By the method explained in the previous section we can directly compute the operator corresponding to the function

$$
\lambda^{\vee}=\frac{\sigma^{\vee}\left(z-h_{1} e_{1}\right) \sigma^{\vee}\left(z+h_{1} e_{1}\right)}{\left(\sigma^{\vee}(z)\right)^{2}}=\frac{\left(\left(z_{1}-h_{1}\right)^{3} / 3-z_{2}\right)\left(\left(z_{1}+h_{1}\right)^{3} / 3-z_{2}\right)}{\left(z_{1}^{3} / 3-z_{2}\right)^{2}} .
$$

For simplicity we put $h_{1}=h_{2}=1, x=0, \beta=(1,1 / 3)$. We have

$$
\begin{aligned}
& L_{11}^{\vee}\left(\lambda^{\vee}\right)=v_{20} T_{1}^{2}+v_{11} T_{1} T_{2}+v_{1} T_{1}, \quad L_{12}^{\vee}\left(\lambda^{\vee}\right)=u_{1} T_{1}, \\
& u_{1}=\frac{-2 n_{1}^{2}\left(n_{1}+1\right)\left(n_{1}+2\right)\left(n_{1}\left(n_{1}+3\right)+5\right)+6 n_{2}\left(2 n_{1}\left(n_{1}+1\right)\left(n_{1}+2\right)-3\right)-18 n_{2}^{2}}{\left(n_{1}+2\right)\left(6 n_{2}+n_{1}\left(n_{1}\left(n_{1}+6\right)+13\right)+14\right)} \text {, } \\
& v_{20}=-u_{1}-\frac{n_{1}}{n_{1}+2}, \quad v_{11}=\frac{\left(n_{1}+2\right)\left(2 n_{1}\left(n_{1}+1\right)-u_{1}\left(n_{1}+3\right)\right)-6 n_{2}}{3\left(n_{1}+2\right)\left(n_{1}+1\right)}, \\
& v_{1}=2-v_{11}-\frac{2}{n_{1}+2} \\
& L_{21}^{\vee}\left(\lambda^{\vee}\right)=q_{30} T_{1}^{3}+q_{21} T_{1}^{2} T_{2}+q_{12} T_{1} T_{2}^{2}+q_{20} T_{1}^{2}+q_{11} T_{1} T_{2}+q_{1} T_{1}, \\
& L_{22}^{\vee}\left(\lambda^{\vee}\right)=p_{20} T_{1}^{2}+p_{11} T_{1} T_{2}+p_{1} T_{1}, \\
& p_{1}=\frac{2\left(n_{1}^{6}+9 n_{1}^{5}+37 n_{1}^{4}+48+n_{1}^{2}\left(106-27 n_{2}\right)\right)}{3\left(n_{1}+2\right)\left(n_{1}^{3}+6 n_{1}^{2}+13 n_{1}+6 n_{2}+14\right)} \\
& +\frac{2\left(n_{1}\left(88-21 n_{2}\right)+n_{1}^{3}\left(83-6 n_{2}\right)+21 n_{2}+9 n_{2}^{2}\right)}{3\left(n_{1}+2\right)\left(n_{1}^{3}+6 n_{1}^{2}+13 n_{1}+6 n_{2}+14\right)},
\end{aligned}
$$




$$
\begin{aligned}
p_{11}= & \frac{2\left(5+n_{1}\left(11+n_{1}\left(n_{1}+6\right)\right)-3 n_{2}\right)-9\left(n_{1}+1\right)\left(n_{1}+2\right) q_{12}}{3\left(n_{1}+2\right)\left(n_{1}+3\right)}, \\
q_{1}= & \frac{3 p_{12}+3 p_{1}-4+n_{1}\left(p_{11}+p_{1}-2\right)}{3\left(n_{1}+1\right)}+q_{12}, \\
p_{20}= & \frac{-2 n_{1}^{6}-24 n_{1}^{5}-123 n_{1}^{4}+12 n_{1}^{3}\left(n_{2}-28\right)}{\left(n_{1}+3\right)\left(n_{1}^{3}+9 n_{1}^{2}+28 n_{1}+6 n_{2}+34\right)} \\
& +\frac{n_{1}^{2}\left(72 n_{2}-501\right)+6 n_{1}\left(21 n_{2}-64\right)-18\left(n_{2}^{2}-2 n_{2}+7\right)}{\left(n_{1}+3\right)\left(n_{1}^{3}+9 n_{1}^{2}+28 n_{1}+6 n_{2}+34\right)}, \\
q_{11}= & -q_{1}-q_{12}, \quad q_{20}=\frac{3\left(n_{1}+1\right)\left(q_{12}-q_{1}\right)}{n_{1}+3}-q_{21}, \quad q_{30}=\frac{2}{n_{1}+3}-p_{20}-1, \\
q_{21}= & \frac{9\left(n_{1}\left(n_{1}\left(n_{1}+3\right)+3\right)-3 n_{2}-5\right) q_{12}+\left(\left(n_{1}+3\right)^{3}-3 n_{2}\right) q_{30}}{3\left(5+n_{1}\left(n_{1}\left(n_{1}+6\right)+12\right)-3 n_{2}\right)}, \\
q_{12}= & \frac{2\left(46+61 n_{1}^{4}+12 n_{1}^{5}+n_{1}^{6}+n_{1}\left(161-66 n_{2}\right)\right)}{9\left(n_{1}+2\right)\left(n_{1}^{3}+6 n_{1}^{2}+13 n_{1}+20\right)} \\
& +\frac{2\left(n_{1}^{3}\left(163-6 n_{2}\right)-21 n_{2}+9 n_{2}^{2}-4 n_{1}^{2}\left(9 n_{2}-58\right)\right)}{9\left(n_{1}+2\right)\left(n_{1}^{3}+6 n_{1}^{2}+13 n_{1}+20\right)} .
\end{aligned}
$$

Similarly, for the function

$$
\mu^{\vee}=\frac{\sigma^{\vee}\left(z-h_{2} e_{2}\right) \sigma^{\vee}\left(z+h_{2} e_{2}\right)}{\left(\sigma^{\vee}(z)\right)^{2}}=\frac{\left(z_{1}^{3} / 3-\left(z_{2}-h_{2}\right)\right)\left(z_{1}^{3} / 3-\left(z_{2}+h_{2}\right)\right)}{\left(z_{1}^{3} / 3-z_{2}\right)^{2}}
$$

we have

$$
\begin{aligned}
& L_{11}^{\vee}\left(\mu^{\vee}\right)=f_{11} T_{1} T_{2}+f_{02} T_{2}^{2}+f_{2} T_{2}, \quad L_{12}^{\vee}\left(\mu^{\vee}\right)=g_{2} T_{2}, \\
& f_{11}=\frac{18 n_{1}}{n_{1}\left(n_{1}\left(n_{1}+3\right)+4\right)+6\left(n_{2}+2\right)}, \quad f_{02}=\frac{f_{11}\left(n_{1}+2\right)}{3 n_{1}}-1 \text {, } \\
& f_{2}=1-f_{02}, \quad g_{2}=-f_{11}, \\
& L_{21}^{\vee}\left(\mu^{\vee}\right)=r_{21} T_{1}^{2} T_{2}+r_{12} T_{1} T_{2}^{2}+r_{03} T_{2}^{3}+r_{11} T_{1} T_{2}+r_{02} T_{2}^{2}+r_{2} T_{2}, \\
& L_{22}^{\vee}\left(\mu^{\vee}\right)=j_{11} T_{1} T_{2}+j_{02} T_{2}^{2}+j_{2} T_{2}, \\
& r_{21}=\frac{18\left(n_{1}+1\right)}{n_{1}^{3}+6 n_{1}^{2}+13 n_{1}+20+6 n_{2}}, \quad r_{03}=\frac{2\left(n_{1}+2\right)}{n_{1}^{3}+3 n_{1}^{2}+4 n_{1}+6\left(n_{2}+2\right)}, \\
& r_{12}=\frac{9 n_{1} r_{03}+9 n_{1}^{2} r_{03}+6 r_{21}+5 n_{1} r_{21}+n_{1}^{2} r_{21}}{3 n_{1}^{2}+9 n_{1}+6}, \\
& r_{11}=\frac{-n_{1}^{3} r_{12}-6\left(n_{2}+2\right) r_{12}+n_{1}^{2}\left(9 r_{03}-6 r_{12}+r_{21}\right)+n_{1}\left(3 r_{21}-9 r_{03}-7 r_{12}\right)}{n_{1}^{3}+3 n_{1}^{2}+4 n_{1}+6\left(n_{2}+2\right)}, \\
& r_{02}=-\frac{2\left(n_{1}^{3}+3 n_{1}^{2}+4 n_{1}+15+6 n_{2}\right) r_{03}}{n_{1}^{3}+3 n_{1}^{2}+4 n_{1}+6\left(n_{2}+2\right)}, \quad r_{2}=-r_{02}-r_{03}, \quad j_{11}=-r_{21} \text {, } \\
& j_{02}=-\frac{2+n_{1}+3 n_{1} r_{03}}{n_{1}+2}, \quad j_{2}=\frac{n_{1}+2-j_{02}\left(n_{1}+2\right)-3 n_{1} r_{02}-6 n_{1} r_{03}}{n_{1}+2} .
\end{aligned}
$$

4.4. $2 \times 2$-matrix operators: Rational spectral variety. Let us consider the DBA module $\hat{M}_{\Omega, 1}$ of the case of $\Lambda=1$. We set

$$
G=z_{1} w_{1}+z_{1} w_{2}+z_{2} w_{2}, G_{1}=4 z_{1} w_{1}+2 z_{1} w_{2}+z_{2} w_{2}, G_{2}=z_{1} w_{1}-z_{1} w_{2}+z_{2} w_{2} .
$$


Here $B=1$ in (17) and $c_{1}=2, c_{2}=-1$ in (18). We choose the following basis of $\hat{M}_{\Omega, 1}$ :

$$
\begin{aligned}
& \psi_{1}=\frac{z_{2} w_{1}}{G}\left(\frac{G_{1}}{G}\right)^{n_{1}}\left(\frac{G_{2}}{G}\right)^{n_{2}}, \\
& \psi_{2}=\frac{z_{1} w_{1}+(-1)^{n_{2}} 2^{n_{1}} z_{1} w_{2}+2^{2 n_{1}} z_{2} w_{2}}{G}\left(\frac{G_{1}}{G}\right)^{n_{1}}\left(\frac{G_{2}}{G}\right)^{n_{2}} .
\end{aligned}
$$

We have

$$
\psi_{i}\left(n_{1}, n_{2},[1,0],\left[t_{1}, t_{2}\right]\right)-\psi_{i}\left(n_{1}, n_{2},\left[t_{1}, t_{2}\right],[0,1]\right)=0
$$

Let

These functions satisfy the identity

$$
\lambda_{1}=\frac{z_{2} w_{1}}{G}, \lambda_{2}=\frac{z_{1} z_{2} w_{1} w_{2}}{G^{2}} .
$$

$$
\lambda_{i}\left([1,0],\left[t_{1}, t_{2}\right]\right)-\lambda_{i}\left(\left[t_{1}, t_{2}\right],[0,1]\right)=0 .
$$

It is easy to check that

$$
\left(\begin{array}{cc}
T_{1}+a_{2} T_{2}+a & b_{1} T_{1}+b_{2} T_{2}+b \\
c_{2} T_{2}+c & d_{1} T_{1}+d_{2} T_{2}+d
\end{array}\right)\left(\begin{array}{c}
\psi_{1} \\
\psi_{2}
\end{array}\right)=\lambda_{1}\left(\begin{array}{c}
\psi_{1} \\
\psi_{2}
\end{array}\right)
$$

where

$$
\begin{aligned}
& a_{2}=-1+\left(-2+(-1)^{n_{2}} 2^{n_{1}}+3 \cdot 2^{1+2 n_{1}}\right) b_{1}, \quad a=-4-a_{2}-b_{1}, \\
& b_{1}=\frac{3}{-1+4^{1+n_{1}}}, \quad b_{2}=\frac{3\left(-1+(-1)^{n_{2}} 2^{1+n_{1}}\right)}{\left(1+(-1)^{n_{2}} 2^{n_{1}}\right)\left(-1+4^{1+n_{1}}\right)}, \quad b=-4 b_{1}-b_{2}, \\
& d_{1}=\frac{-1+4^{n_{1}}}{-1+4^{1+n_{1}}}, \quad c_{2}=\frac{1}{2}\left(1-(-1)^{n_{2}} 2^{n_{1}}\right)+\left(-2+(-1)^{n_{2}} 2^{n_{1}}+3 \cdot 2^{1+2 n_{1}}\right) d_{1}, \\
& c=1-c_{2}-d_{1}, \quad d_{2}=\frac{\left(-1+(-1)^{n_{2}} 2^{1+n_{1}}\right) d_{1}}{1+(-1)^{n_{2}} 2^{n_{1}}}, \quad d=-4 d_{1}-d_{2} .
\end{aligned}
$$

In a similar way we get

$$
\left(\begin{array}{ll}
L_{11} & L_{12} \\
L_{21} & L_{22}
\end{array}\right)\left(\begin{array}{l}
\psi_{1} \\
\psi_{2}
\end{array}\right)=\lambda_{2}\left(\begin{array}{l}
\psi_{1} \\
\psi_{2}
\end{array}\right)
$$

$L_{11}=-\frac{1}{2} T_{1} T_{2}+a_{22}^{\prime} T_{2}^{2}+\frac{1}{2} T_{1}+a_{2}^{\prime} T_{2}+a^{\prime}$,

$L_{12}=b_{12}^{\prime} T_{1} T_{2}+b_{22}^{\prime} T_{2}^{2}+b_{1}^{\prime} T_{1}+b_{2}^{\prime} T_{2}+b^{\prime}$,

$L_{21}=c_{22}^{\prime} T_{2}^{2}+c_{2}^{\prime} T_{2}+c^{\prime}, \quad L_{22}=d_{12}^{\prime} T_{1} T_{2}+d_{22}^{\prime} T_{2}^{2}+d_{1}^{\prime} T_{1}+T_{2}+d^{\prime}$,

where

$$
\begin{aligned}
a_{22}^{\prime}= & \frac{1}{2}\left(1-4\left(1+(-1)^{n_{2}} 2^{1+3 n_{1}}-3 \cdot 4^{n_{1}}\right) b_{12}^{\prime}+(-1)^{n_{2}} 2^{n_{1}} b_{2}^{\prime}-(-1)^{n_{2}} 8^{n_{1}} b_{2}^{\prime}\right), \\
a_{2}^{\prime}= & \left(2-2 a_{22}^{\prime}-b_{12}^{\prime}-2(-1)^{n_{2}} 2^{n_{1}} b_{12}^{\prime}\right), \quad a^{\prime}=-a_{2}^{\prime}-a_{22}^{\prime}, \quad b_{12}^{\prime}=\frac{3}{2\left(-1+4^{1+n_{1}}\right)}, \\
b_{22}^{\prime}= & \frac{1}{2}\left(-4\left(1+(-1)^{n_{2}} 2^{1+n_{1}}\right) b_{12}^{\prime}-\left(1+(-1)^{n_{2}} 2^{n_{1}}\right) b_{2}^{\prime}\right), \quad b_{1}^{\prime}=-b_{12}^{\prime}, \\
b_{2}^{\prime}= & \frac{3}{-1+4^{n_{1}}}, \quad b^{\prime}=-b_{2}^{\prime}-b_{22}^{\prime}, \\
c_{22}^{\prime}= & -\frac{1}{4}\left(-1+(-1)^{n_{2}} 2^{n_{1}}\right)\left(-1+\left(-8-(-1)^{n_{2}} 2^{3+n_{1}}+4^{2+n_{1}}\right) d_{12}^{\prime}\right. \\
& \left.+(-1)^{n_{2}} 2^{1+n_{1}}\left(1+(-1)^{n_{2}} 2^{n_{1}}\right)\right),
\end{aligned}
$$




$$
\begin{aligned}
& c_{2}^{\prime}=-\frac{1}{2}-2 c_{22}^{\prime}-\left(1+(-1)^{n_{2}} 2^{1+n_{1}}\right) d_{12}^{\prime}, \quad c^{\prime}=-c_{2}^{\prime}-c_{22}^{\prime}, \quad d_{1}^{\prime}=-d_{12}^{\prime}, \\
& d_{12}^{\prime}=\frac{-1+4^{n_{1}}}{2\left(-1+4^{1+n_{1}}\right)}, \quad d^{\prime}=-1-d_{22}^{\prime} \\
& d_{22}^{\prime}=\frac{1}{2}\left(-4\left(1+(-1)^{n_{2}} 2^{1+n_{1}}\right) d_{12}^{\prime}-\left(1+(-1)^{n_{2}} 2^{n_{1}}\right)\right) .
\end{aligned}
$$

\section{ACKNOWLEDGMENTS}

This research was partially supported by JSPS Grant-in-Aid for Scientific Research (C) 23540245. The first author was also partially supported by RFBR grant 11-01-12106ofi-m-2011. The second author would like to thank Koji Cho for useful suggestions on the proofs of theorems. A part of this work was done while the the first author stayed at Tsuda College. He is grateful to this institution for kind hospitality. The authors are also grateful to the referee for a number of useful comments that improved the paper.

\section{REFERENCES}

[1] I.M. Krichever and S.P. Novikov, Two dimensionalized Toda lattice, commuting difference operators, and holomorphic bundles, Russian Math. Surveys, 58:3 (2003), 473-510. MR 1998774(2004j:37144)

[2] I.M. Krichever, Algebraic curves and non-linear difference equations. Russian Math. Surveys, 33:4 (1978), 255-256. MR510681 (80k:58055)

[3] D. Mumford, An algebro-geometric construction of commuting operators and of solutions to the Toda lattice equation, Korteweg de Vries equation and related non-linear equations, Proc. Int. Symp. on Alg. Geom. (Kyoto Univ., Kyoto, 1977), Kinokuniya, Tokyo, 1978, 115-153. MR578857 (83j:14041)

[4] A.E. Mironov, Discrete analogues of Dixmier operators, Sbornik: Mathematics, 198:10 (2007), 1433-1442. MR2362822(2008j:37151)

[5] I.M. Krichever, Methods of algebraic geometry in the theory of non-linear equations, Russian Math. Surveys, 32:6 (1977), 32:6, 185-213.

[6] A.B. Zheglov, On rings of commuting partial differential operators, arXiv:1106.0765 (to appear in St. Petersburg Math. J.).

[7] H. Kurke, D. Osipov and A. Zheglov, Commuting differential operators and higher-dimensional algebraic varieties, arXiv:1211.0976.

[8] A. Nakayashiki, Structure of Baker-Akhiezer modules of principally polarized Abelian varieties, commuting partial differential operators and associated integrable systems, Duke Math. J. 62 (1991), 315-358. MR 1104527 (92j:14056)

[9] A. Nakayashiki, Commuting partial differential operators and vector bundles over Abelian varieties, Amer. J. Math. 116 (1994), 65-100. MR1262427 (95j:14063)

[10] T. Miwa, On Hirota's difference equations, Proc. Japan Acad. Ser. A 58 (1982), 9-12. MR649054 (83f:58042)

[11] E. Date, M. Jimbo and T. Miwa, Method for generating discrete soliton equations I, J. Phys. Soc. Japan 51-12 (1982), 4116-4124, ibid. II, J. Phys. Soc. Japan 51-12 (1982), 4125-4131, ibid. III, J. Phys. Soc. Japan 52-2 (1983), 388-393, ibid. IV, J. Phys. Soc. Japan 52-3 (1983), 761-765,ibid. V, J. Phys. Soc. Japan 52-3 (1983), 766-771.

[12] I.A. Melnik and A.E. Mironov, Baker-Akhiezer Modules on Rational Varieties, SIGMA 6 (2010), 030, 15 pages. MR2647309 (2011d:14067)

[13] A. Nakayashiki, On hyperelliptic abelian function of genus 3, J. Geometry and Physics 61 (2011), 961-985. MR2782474 (2012k:14042)

[14] F. Klein, Ueber hyperelliptische Sigmafunctionen, Math. Ann. 27 (1886), 341-464. MR.1510386

[15] F. Klein, Ueber hyperelliptische Sigmafunctionen (Zweiter Aufsatz), Math. Ann. 32 (1888), 351380. MR 1510518

[16] V. M. Buchstaber, V. Z. Enolski and D. V. Leykin, Rational analogue of Abelian functions, Funct. Annal. Appl. 33-2 (1999), 83-94. MR1719334(2000i:14051)

[17] A. Nakayashiki, Algebraic expressions of sigma functions of $(n, s)$ curves, Asian J. Math. 14-2 (2010), 175-212. MR2746120(2011k:14029) 
Sobolev Institute of Mathematics, 630090 Novosibirsk, Russia - and — Laboratory of Geometric Methods in Mathematical Physics, Moscow State University

E-mail address: mironov@math.nsc.ru

Department of Mathematics, Tsuda College, Kodaira, Tokyo, Japan

E-mail address: atsushi@tsuda.ac.jp

Originally published in English 